\title{
Predictions for LHC from SO(4) MWT
}

\section{Ari Hietanen*}

E-mail: hietanen@cp3-origins.net

\section{Claudio Pica}

E-mail: pica@cp3-origins.net

\section{Francesco Sannino}

E-mail: sannino@cp3-origins.net

\section{Ulrik Søndergaard}

E-mail: sondergaardecp3-origins. net

$C P^{3}$-Origins \& the Danish Institute for Advanced Study DIAS, University of Southern Denmark, Campusvej 55, DK-5230 Odense M, Denmark.

We investigate the vector, axial and pseudo scalar mass spectrum of an $\mathrm{SO}(4)$ - MWT gauge theory with fermions in the vector representation of $\mathrm{SO}(4)$. Here we present the preliminary lattice results for the masses of vector and axial vector meson using Wilson fermions. These spectra are crucial for the discovery and to guide the searches of composite dynamics at the LHC.

CP3-Origins-2013-045 DNRF90 \& DIAS-2013-45

31st International Symposium on Lattice Field Theory - LATTICE 2013

July 29 - August 3, 2013

Mainz, Germany

${ }^{*}$ Speaker. 


\section{Introduction}

In Technicolor (TC) theories a new a strongly interacting gauge theory is responsible for the electroweak symmetry breaking phenomenon. This scenario leads to a spectrum of composite particles with masses in the $\mathrm{TeV}$ energy range. The specific spectral properties of the composite states can be calculated via lattice simulations and depend on the specific underlying gauge theory. The first general classification of $\mathrm{SU}(N)$ gauge theories that can lead to phenomenologically acceptable composite electroweak theories appeared in $[1,2,3]$, including the presence of a light composite Higgs-like state $[4,5,6]$. The generalization to SO, Sp and exceptional groups appeared in [7, 8].

Furthermore it has been recently argued that a phenomenologically viable TC Higgs mass can arise via a cancellation between the intrinsic positive mass squared of the composite state and the large negative top corrections [9]. It is relevant to test these expectations via lattice simulations. However the isosinglet scalar meson is challenging to measure on lattice because of noisy disconnected diagrams, and furthermore one should add four-fermion interactions allowing the generation of the top mass operator. It is for these reasons that we turn our attention first to the spectrum of isotriplet spin-one resonances whose spectral properties can be determined more reliably via lattice simulations, and furthermore, their masses are expected to remain close to the one directly determined via lattice simulations, in units of the electroweak scale (i.e. pion decay constant).

In this proceedings we provide preliminary results for the physical values of vector and axial vector meson from the $\mathrm{SO}(4)$ vector Minimal Walking Theory (MWT) at a fixed lattice spacing. Earlier data are already published in $[10,11]$. The $S O(4)$ vector MWT is phenomenologically interesting theory for several reasons. First, from perturbative arguments the theory is expected to break chiral symmetry, but to be near the lower bound of conformal window [7]. Second, the theory contains possibly light composite dark matter particles. These new light states emerge because the techni-fermions belong to a real representation, and therefore the chiral symmetry breaking pattern is expected to be $\mathrm{SU}(4) \rightarrow \mathrm{SO}(4)$. This leads to nine Goldstone bosons. Three of these are eaten by the SM gauge bosons leaving six additional Goldstone bosons forming an electroweak complex triplet. The neutral isospin zero component is a possible dark matter candidate called ITIMP [14]. Third, compared to the SU(2) adj-MWT using the standard hyper charge assignments there are no fractionally charged composite particles as technigluons cannot form bound states with a techniquark. The spectrum of the TC theories have also been studied in $[12,13]$ and theories with composite dark matter in $[15,16,17]$.

\section{SO(4) gauge theory with two fundamental on the Lattice}

We used, in our simulations, the Wilson plaquette action with unimproved Wilson fermions

$$
S=S_{\mathrm{F}}+S_{\mathrm{G}}
$$

where

$$
S_{\mathrm{G}}=\beta \sum_{x} \sum_{\mu, v<\mu}\left[1-\frac{1}{N_{\mathrm{c}}} \operatorname{Tr} U_{\mu v}(x)\right]
$$

is the Yang-Mills gauge action. $U_{\mu v}(x)$ is the plaquette defined in terms of the link variables as

$$
U_{\mu v}(x)=U_{\mu}(x) U_{v}(x+\hat{\mu}) U_{\mu}^{T}(x+\hat{\mu}+\hat{v}) U_{v}^{T}(x+\hat{v}) .
$$




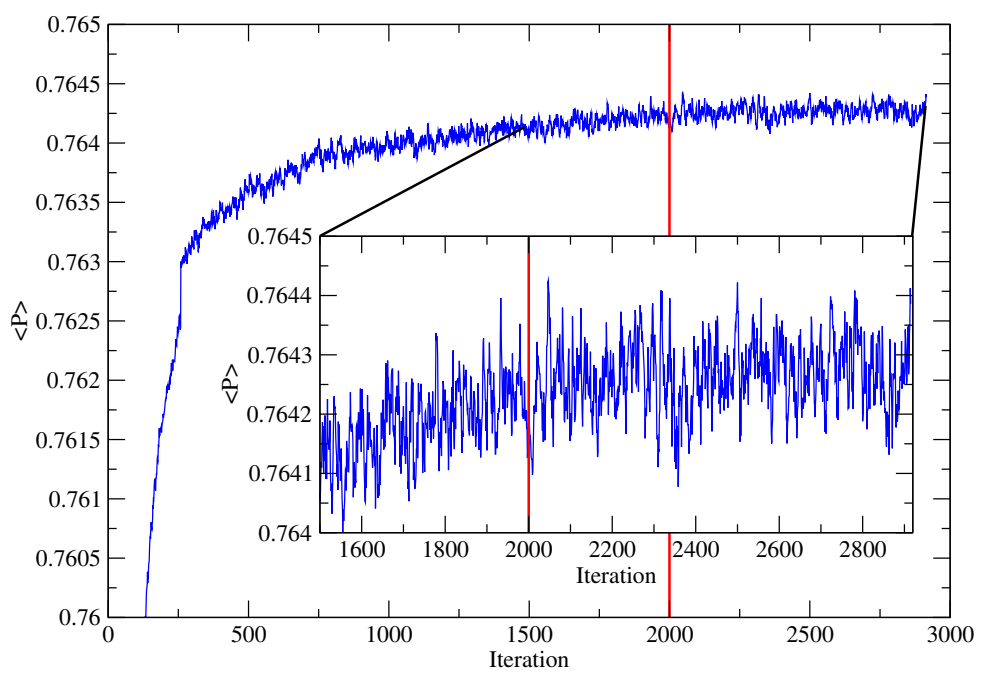

Figure 1: Thermalization of Plaquette expectation values when starting from a random configurations. The first 2000 configurations have been discarded in the analysis.

The Wilson fermion action is

$$
S_{\mathrm{F}}=\sum_{f} \sum_{x, y} \bar{\psi}_{f}(x) M(x, y) \psi_{f}(y)
$$

with $f$ running over fermion flavors and the Wilson-Dirac matrix $M(x, y)$ given by

$$
\sum_{y} M(x, y) \psi(y)=\left(4+m_{0}\right) \psi(x)-\frac{1}{2} \sum_{\mu}\left[\left(1+\gamma_{\mu}\right) U_{\mu}^{T}(x-\hat{\mu}) \psi(x-\hat{\mu})+\left(1-\gamma_{\mu}\right) U_{\mu}(x) \psi(x+\hat{\mu})\right] .
$$

The observables we are interested in are the PCAC mass, pseudo scalar meson, vector meson, and axial vector meson mass as well as the pseudo scalar decay constant defined in [10].

\section{Lattice Results}

In this proceedings we present the preliminary results for one lattice spacing $\beta=7$ in the chiral region for $V=64 \times 32^{3}$. Compared to the results at smaller volumes $V=64 \times 24^{3}$ [10] we observe considerable finite volume effects. However, the conclusion suggesting the occurrence of chiral symmetry breaking is unaffected.

The configurations for the heavier masses were generated using standard HMC algorithm. For the two points closest to the chiral limit Hasenbusch preconditioning was used [18]. The trajectory length was chosen to be one. The thermalization of the simulations is slow and takes about 2000 iterations. An example of thermalization of Plaquette is shown in Fig. 1 for $m_{0}=-0.3$. Other quantities have similar long tails, approaching the correct value slowly. The auto correlation time is about 10 iterations.

In Fig. 2 we plot the masses of the pseudo scalar, vector, and axial vector mesons. The three lightest data points have low statistics with the shown statistical errorbars probably underestimates. 


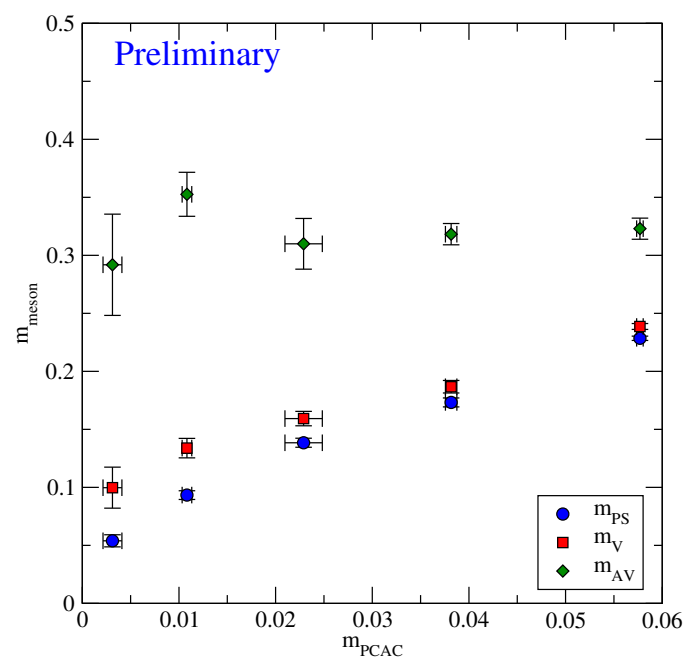

Figure 2: The masses of pseudo scalar, vector, and axial vector meson as a function of PCAC-quark mass.
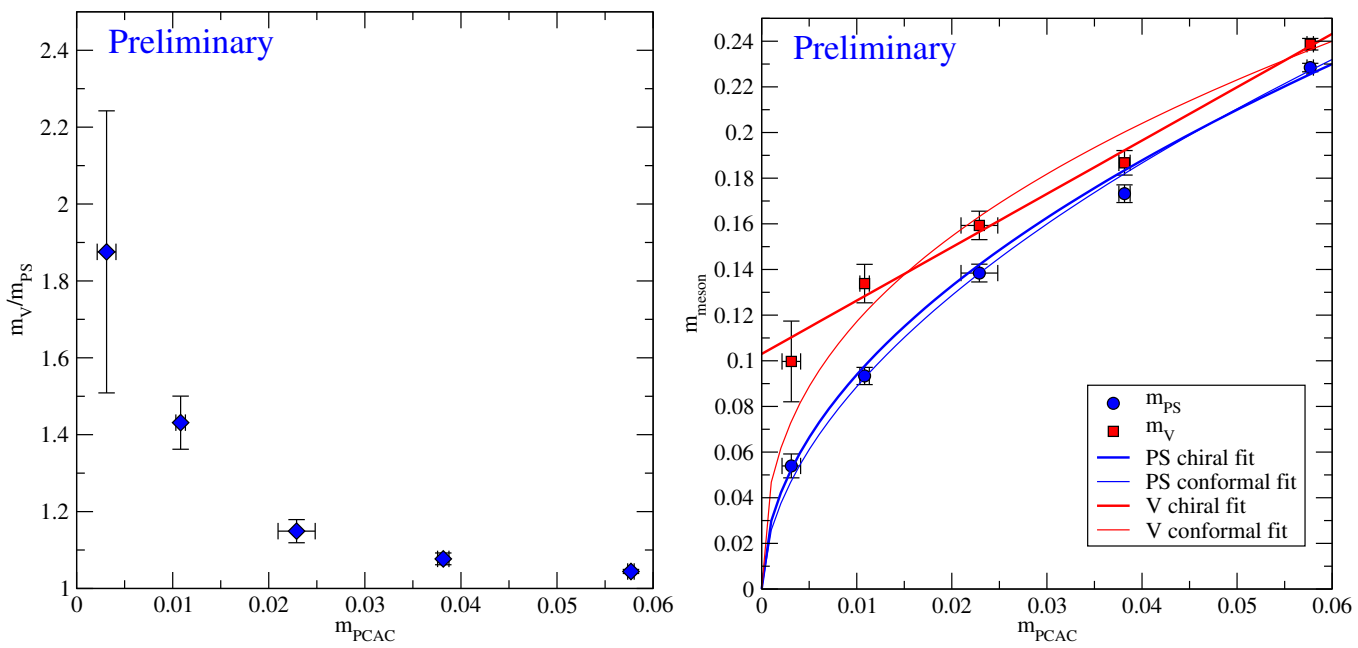

Figure 3: Left: Ratio between vector and pseudo scalar meson. Right: Chiral (thick lines) and conformal (thin lines) fits to the pseudo scalar and vector meson masses.

However, there is a clear separation between different particle states indicating the occurrence of chiral symmetry breaking. This can also be seen from the left panel of Fig. 3 where we plot the ratio of vector to pseudo scalar meson mass, which diverges in the chiral limit.

For a more systematic comparison between the possible occurrence of chiral symmetry and the hypothesis of large distance conformality, we perform also a conformal fit

$$
m_{\mathrm{PS}, \mathrm{V}, \mathrm{AV}}=A m_{\mathrm{q}}^{\frac{1}{1+\gamma}},
$$

where $0 \leq \gamma \leq 2$ is a universal exponent. The chiral symmetry breaking scenario would, on the other hand, predict the following relations among the measured hadron masses as function of the 


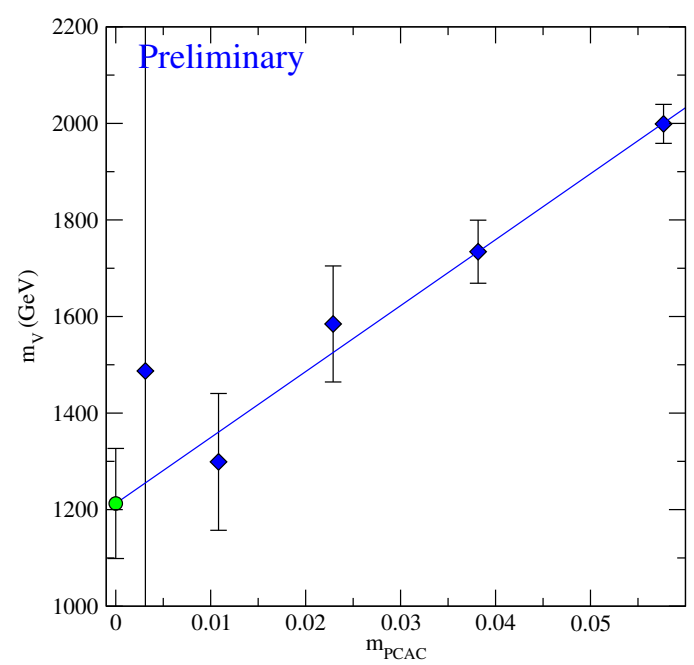

Figure 4: Chiral extrapolation of vector meson mass in physical units: $m_{v}=m_{\mathrm{V}}^{\text {lat }} / f_{\mathrm{PS}}^{\text {lat }} 246 \mathrm{GeV}$.

\begin{tabular}{|c|c|c|c|}
\hline Meson & Fit function & Best parameter & $\chi^{2} /$ dof \\
\hline PS ChSB & $A \sqrt{m}$ & $A=0.939(7)$ & $11.7 / 4$ \\
\hline PS conformal & $A m^{\frac{1}{1+\gamma}}$ & $\begin{array}{l}A=1.05(6) \\
\gamma=0.86(4)\end{array}$ & $7.2 / 3$ \\
\hline Vector ChSB & $A+B m$ & $\begin{array}{l}A=0.103(7) \\
B=2.34(12)\end{array}$ & $2.1 / 3$ \\
\hline Vector conformal & $A m^{\frac{1}{1+\gamma}}$ & $\begin{array}{l}\gamma=1.50(7) \\
A=0.74(6)\end{array}$ & $12.1 / 3$ \\
\hline Axial vector ChSB & $A+B m$ & $\begin{array}{l}A=0.103(7) \\
B=2.34(12)\end{array}$ & $2.1 / 3$ \\
\hline Axial Vector conformal & $A m^{\frac{1}{1+\gamma}}$ & $\begin{array}{l}\gamma=2 \\
A=0.924(17)\end{array}$ & $93.3 / 3$ \\
\hline
\end{tabular}

Table 1: Different types of fit functions in the chiral regime for the data with $m$ identified with the $m_{\mathrm{PCAC}}$.

quark mass:

$$
\begin{aligned}
\frac{m_{\mathrm{PS}}^{2}}{m_{\mathrm{q}}} & =A+\mathscr{O}\left(m_{\mathrm{q}} \log \left(m_{\mathrm{q}}\right)\right) \\
m_{\mathrm{V}, \mathrm{AV}} & =A+B m_{\mathrm{q}}+\mathscr{O}\left(m_{\mathrm{q}} \log \left(m_{\mathrm{q}}\right)\right) .
\end{aligned}
$$

The different parameters and $\chi^{2} /$ dof for the fits are given in the Table 1 . The scenario of chiral symmetry breaking seems favored. The axial vector meson dependence on the underlying fermion mass is, for example, clearly incompatible with the conformal fit. The different fits for pseudo scalar and vector meson masses are represented in the right panel of Fig. 3.

To determine the physical values of the masses we set the pseudo scalar decay constant to $246 \mathrm{GeV}$ to recover the physical weak gauge boson masses. After performing a chiral extrapolation 
with these values we obtain a mass of $m_{V}=1.21(11) \mathrm{TeV}$, as shown in Fig. 4. Similarly for the axial mass we have $m_{A}=3.5(3) \mathrm{TeV}$. Potentially large unknown corrections from the continuum extrapolation could arise requiring, in the future, to provide new measurements at another value of $\beta$. In addition, we still need the renormalization constant $Z_{a}$ which enters in the denominator of the pseudo scalar decay constant. The value from perturbation theory for $Z_{a}$ in $\operatorname{SO}(N)$ gauge theories with vector representation fermions is given in [19]

$$
Z_{a}=1-\frac{g_{0}^{2}}{16 \pi^{2}} \frac{N-1}{2} 15.7 \stackrel{N=4}{=} 1-1.1931 / \beta \stackrel{\beta=7}{=} 0.823 .
$$

The perturbative correction would increase the physical vector meson mass to $1.47(14) \mathrm{TeV}$.

\section{Summary}

We have presented preliminary results of the simulations of the SO(4)-MWT with $\beta=7$ and $V=64 \times 32^{3}$. The data seem to strongly suggest the occurrence of chiral symmetry breaking. In addition, we have also obtained a preliminary predictions for the vector and axial vector meson masses. The vector meson mass $1.47(14) \mathrm{TeV}$ is quite close to that one of the $\rho$ meson in QCD obtained by setting $f_{\pi}=246 \mathrm{GeV}$ where as the axial vector mass is much heavier. Such a vector meson mass should be within LHC experimental reach. However, although intriguing, these results need further confirmation by going beyond the one lattice spacing approximation. At the moment we are improving the results by performing more simulations near the chiral limit as well as with an another lattice spacing.

\section{Acknowlegements}

This work was supported by the Danish National Research Foundation DNRF:90 grant and by a Lundbeck Foundation Fellowship grant. We also acknowledge PRACE for awarding us access to resource on MARENOSTRUM based in Spain at BSC. Additional computing facilities were provided by the Danish Centre for Scientific Computing.

\section{References}

[1] F. Sannino and K. Tuominen, Phys. Rev. D 71, 051901 (2005) [hep-ph/0405209].

[2] D. D. Dietrich and F. Sannino, Phys. Rev. D 75, 085018 (2007) [hep-ph/0611341].

[3] C. Pica and F. Sannino, Phys. Rev. D 83, 035013 (2011) [arXiv:1011.5917 [hep-ph]].

[4] F. Sannino and M. Shifman, Phys. Rev. D 69, 125004 (2004) [hep-th/0309252].

[5] D. K. Hong, S. D. H. Hsu and F. Sannino, Phys. Lett. B 597, 89 (2004) [hep-ph/0406200].

[6] D. D. Dietrich, F. Sannino and K. Tuominen, Phys. Rev. D 72, 055001 (2005) [hep-ph/0505059].

[7] F. Sannino, Phys. Rev. D 79, 096007 (2009) [arXiv:0902.3494 [hep-ph]].

[8] M. Mojaza, C. Pica, T. A. Ryttov and F. Sannino, Phys. Rev. D 86, 076012 (2012) [arXiv:1206.2652 [hep-ph]].

[9] R. Foadi, M. T. Frandsen and F. Sannino, Phys. Rev. D 87, 095001 (2013) [arXiv:1211.1083 [hep-ph]]. 
[10] A. Hietanen, C. Pica, F. Sannino and U. I. Sondergaard, Phys. Rev. D 87, no. 3, 034508 (2013) [arXiv:1211.5021 [hep-lat]].

[11] A. Hietanen, C. Pica, F. Sannino and U. I. Sondergaard, PoS LATTICE 2012, 065 (2012) [arXiv:1211.0142 [hep-lat]].

[12] Z. Fodor, K. Holland, J. Kuti, D. Nogradi, C. Schroeder, K. Holland, J. Kuti and D. Nogradi et al., Phys. Lett. B 703, 348 (2011) [arXiv:1104.3124 [hep-lat]].

[13] Z. Fodor, K. Holland, J. Kuti, D. Nogradi, C. Schroeder and C. H. Wong, Phys. Lett. B 718, 657 (2012) [arXiv:1209.0391 [hep-lat]].

[14] M. T. Frandsen and F. Sannino, Phys. Rev. D 81, 097704 (2010) [arXiv:0911.1570 [hep-ph]].

[15] R. Lewis, C. Pica and F. Sannino, Phys. Rev. D 85, 014504 (2012) [arXiv:1109.3513 [hep-ph]].

[16] T. Appelquist, R. C. Brower, M. I. Buchoff, M. Cheng, S. D. Cohen, G. T. Fleming, J. Kiskis and M. F. Lin et al., Phys. Rev. D 88, 014502 (2013) [arXiv:1301.1693 [hep-ph]].

[17] A. Hietanen, R. Lewis, C. Pica and F. Sannino, arXiv:1308.4130 [hep-ph].

[18] M. Hasenbusch, Phys. Lett. B 519, 177 (2001) [hep-lat/0107019].

[19] L. Del Debbio, M. T. Frandsen, H. Panagopoulos and F. Sannino, JHEP 0806, 007 (2008) [arXiv:0802.0891 [hep-lat]]. 